\title{
Free Fatty Acids and Stroke From Atrial Fibrillation
}

Can. J. Neurol. Sci. 2011; 38: 803

In this issue of CJNS, Seo et $\mathrm{al}^{1}$ provide evidence that levels of free fatty acids are related to cardioembolic stroke, particularly in atrial fibrillation (AF). Their paper is important, because it is based on a fairly large group $(n=715)$ of well characterized stroke patients from a university hospital stroke registry, admitted between July 2007 and December 2009. The importance of clearly distinguishing stroke subtypes was highlighted in a 2010 meta-analysis ${ }^{2}$ showing that vitamin E probably reduces the risk of ischemic stroke, but increases the risk of hemorrhagic stroke, so lumping all strokes together obscured the effects.

Cardioembolic stroke represented $15 \%$ of cases; of these $72 \%$ were attributed to atrial fibrillation, $9.8 \%$ to recent myocardial infarction, $8.4 \%$ to a patent foramen ovale, $8.4 \%$ from valvular disease, and the rest to less common cardiac problems. Cardioembolic patients had 1.5-fold higher levels of free fatty acids (FFA) compared to non-cardioembolic patients, and among the cardioembolic cases, patients with AF had 1.66-fold higher levels of FFA compared to non-AF cases, whose levels were slightly lower than those of non-cardioembolic cases. Patients with AF whose strokes were attributed to non-cardioembolic causes had similar levels of FFA to AF patients with cardioembolic stroke. That finding may have led the authors to their focus on the effects of FFA on arrhythmia.

Besides effects on arrhythmia, however, there are other ways in which FFA could increase the risk of cardioembolic stroke. Free fatty acids increase oxidative stress $^{3}$, and are involved in post-prandial hypercoagulability; they increase fibrinogen ${ }^{4}$ and Factor VII ${ }^{5,6}$, and reduce fibrinolysis ${ }^{7,8}$. The particular susceptibility of stroke from AF to hypercoagulability is shown by the more than 4-fold increase of stroke in patients with atrial fibrillation and elevated levels of homocysteine ${ }^{9,10}$. The findings in the paper by Seo et al were based, however, on fasting levels of FFA's; the main effects of FFAs are probably in the postprandial state. A further complicating factor is that total FFA's were measured, and FFA's are not all the same.

Free fatty acids are carboxylic acids with a long unbranched (aliphatic) side chain. They may be saturated (e.g. palmitic and stearic acids) or unsaturated (e.g. linoleic and eicosopentanoic acids), with one or more double bonds between carbon atoms, and they may be in cis or trans forms (adjacent hydrogen atoms on the same side or the opposite side of the double bond, respectively). Trans fatty acids, often consumed in hydrogenated vegetable oils, are probably the most harmful macronutrient ${ }^{11}$; even worse than egg yolk, which is clearly harmful ${ }^{12}$. Denmark led the way in banning the sale of foods containing trans fats ${ }^{11}$, and it is high time Canada did so as well. A $2 \%$ increase in energy intake from trans fatty acids increases coronary risk by $23 \%^{11}$. On the other hand, $n-3$ fatty acids, such as $\alpha$-linolenic acid, have beneficial effects.

What can we do about FFAs? The relation of FFAs to insulin resistance, obesity and fatty liver disease suggests that the important roles of weight loss and exercise in stroke prevention ${ }^{13}$ may extend to reducing risk of stroke in atrial fibrillation. It also suggests that the contentious decision to include strokes from atrial fibrillation in the Insulin Resistance Intervention after Stroke (IRIS) trial (http://www.iristrial.org/), a randomized trial of pioglitazone versus placebo in secondary prevention of stroke in patients with insulin resistance, may have been wise. Losing weight, exercising and treating hypertriglyceridemia and insulin resistance may all be ways to reduce the risk of stroke from elevation of FFAs.

J. David Spence
Stroke Prevention \& Atherosclerosis Research Centre
Robarts Research Institute, University of Western Ontario
London, Ontario, Canada

\section{REFERENCES}

1. Seo W-K, Kim J, Kim YH, et al. Elevated free fatty acid is associated with cardioembolic stroke subtype. Can J Neurol Sci. 2011; 38(6):874-9.

2. Schurks M, Glynn RJ, Rist PM, et al. Effects of vitamin E on stroke subtypes: meta-analysis of randomised controlled trials. BMJ. 2010;341:c5702.

3. Soardo G, Donnini D, Domenis L, et al. Oxidative stress is activated by free fatty acids in cultured human hepatocytes. Metab Syndr Relat Disord. 2011;ePub ahead of print May 11, 2011.

4. Pilgeram L. Control of fibrinogen biosynthesis: role of the FFA/albumin ratio. Cardiovasc Eng. 2010;10:78-83.

5. Mutanen M, Freese R. Fats, lipids and blood coagulation. Curr Opin Lipidol. 2001;12:25-9.

6. Miller GJ, Martin JC, Webster J, et al. Association between dietary fat intake and plasma factor VII coagulant activity--a predictor of cardiovascular mortality. Atherosclerosis. 1986;60:269-77.

7. Rigla $\mathrm{M}$, Wagner $\mathrm{AM}$, Borrell $\mathrm{M}$, et al. Postprandial thrombin activatable fibrinolysis inhibitor and markers of endothelial dysfunction in type 2 diabetic patients. Metabolism. 2006;55: 1437-42.

8. Nordoy A, Bonaa KH, Sandset PM, et al. Effect of omega-3 fatty acids and simvastatin on hemostatic risk factors and postprandial hyperlipemia in patients with combined hyperlipemia. Arterioscler Thromb Vasc Biol. 2000;20:259-65.

9. Poli D, Antonucci E, Cecchi E, et al. Culprit factors for the failure of well-conducted warfarin therapy to prevent ischemic events in patients with atrial fibrillation: the role of homocysteine. Stroke. 2005;36:2159-63.

10. Spence JD. Homocysteine-lowering therapy: a role in stroke prevention? Lancet Neurol. 2007;7:830-8.

11. Mozaffarian D, Katan MB, Ascherio A, et al. Trans fatty acids and cardiovascular disease. N Engl J Med. 2006;354:1601-13.

12. Spence JD, Jenkins DJ, Davignon J. Dietary cholesterol and egg yolks: not for patients at risk of vascular disease. Can J Cardiol. 2010;26:e336-e9.

13. Spence JD. Secondary stroke prevention. Nat Rev Neurol. 2010;6: 477-86. 